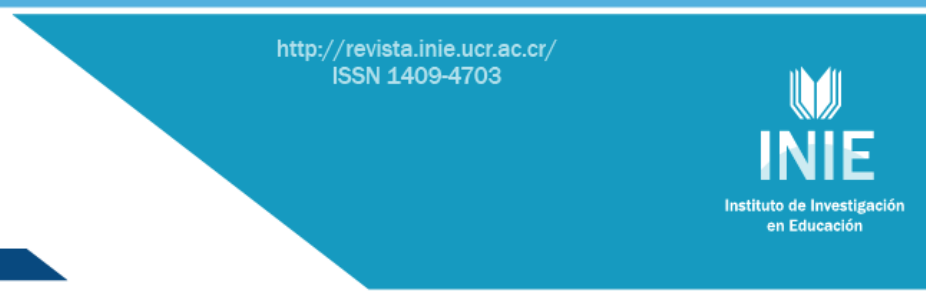

\title{
NECESIDADES DE ORIENTACIÓN DE PADRES Y MADRES DE COLEGIOS ACADÉMICOS DIURNOS DE LA DIRECCIÓN REGIONAL SAN JOSÉ NORTE \\ COUNSELING NEEDS OF PARENTS FROM ACADEMIC DAY SECONDARY SCHOOLS OF THE NORTH SAN JOSÉ REGION
}

\author{
SECCIÓN ESPECIAL \\ Volumen 15, Número 1 \\ Enero - Abril \\ pp. 1-25
}

Este número se publicó el $1^{\circ}$ de enero de 2015
DOI: dx.doi.org/10.15517/aie.v15i1.17630

Roxana Chinchilla Jiménez

Flor Jiménez Segura

Revista indizada en REDALYC, $\underline{\text { SCIELO }}$

Revista distribuida en las bases de datos:

CATÁLOGO DE LATINDEX, IRESIE, CLASE, DIALNET, DOAJ, E-REVIST@S, SHERPA/ROMEO, QUALIS, MIAR

Revista registrada en los directorios:

ULRICH'S, REDIE, RINACE, OEI, MAESTROTECA, PREAL, CLACSO 


\title{
NECESIDADES DE ORIENTACIÓN DE PADRES Y MADRES DE COLEGIOS ACADÉMICOS DIURNOS DE LA DIRECCIÓN REGIONAL SAN JOSÉ NORTE \\ COUNSELING NEEDS OF PARENTS FROM ACADEMIC DAY SECONDARY SCHOOLS OF THE NORTH SAN JOSÉ REGION
}

\author{
Roxana Chinchilla Jiménez ${ }^{1}$ \\ Flor Jiménez Segura ${ }^{2}$
}

\begin{abstract}
Resumen: Este documento emerge de un estudio realizado en el año 2013 y permitió identificar las necesidades de Orientación derivadas del contacto con madres y padres de familia, según las personas profesionales en Orientación. La investigación fue desarrollada desde un paradigma cuantitativo y es de tipo descriptivo y la recopilación de la información se realizó mediante un cuestionario constituido por 15 preguntas abiertas. Algunos de los principales resultados evidencian que las madres y padres desean satisfacer las necesidades básicas de sus hijos e hijas, así como brindarles apoyo en sus estudios, pero no lo logran por situaciones como la pobreza, la falta de empleo y la violencia doméstica. Los Orientadores y las Orientadoras declaran que las madres y los padres necesitan formación en lo referente a reglas claras, manejo de límites y consecuencias. Es necesario que las instituciones educativas promuevan una cultura de acercamiento entre madres $y$ padres con el proceso educativo del estudiantado, con el afán de favorecer el éxito escolar de sus hijos e hijas.
\end{abstract}

Palabras clave: RELACIÓN ESCUELA-FAMILIA, INSTITUCIÓN EDUCATIVA, NECESIDADES DE ORIENTACIÓN, EDUCACIÓN PERMANENTE, COSTA RICA.

\begin{abstract}
This paper arises from a study undertaken in 2013 that allowed the identification of counseling needs of parents, from the perspective of Guidance counselors working in educational institutions. The research is descriptive and was done under a quantitative approach. Information was collected through a 15 open-question questionnaire. Some of the main findings were: that mothers and fathers wish to satisfy their children's basic needs, as well as to support them in school matters, but they are not successful due to poverty, unemployment, and domestic violence. Counselors express that parents need education in relation to clear rules, limit setting, and consequences. It is necessary for schools to promote a culture of involvement of parents with students' educational processes, in order to favor school achievement.
\end{abstract}

Keywords: SCHOOL-FAMILY RELATIONSHIP, EDUCATIONAL INSTITUION COUNSELING NEEDS, LIFE-LONG EDUCATION, COSTA RICA.

\footnotetext{
1 Docente e investigadora de la Escuela de Orientación y Educación Especial y del Instituto de Investigación en Educación, ambos de la Universidad de Costa Rica. Maestría en Terapia Familiar. Licenciada en Ciencias de la Educación con énfasis en Orientación. Dirección electrónica: roxana.chinchilla@ucr.ac.cr

2 Docente e Investigadora de la Escuela de Orientación y Educación Especial y del Instituto de Investigación en Educación. Universidad de Costa Rica. Doctora en Psicología Educativa, Maestría Gerontología, Licda. en Ciencias de la Educación con énfasis en Orientación. Dirección electrónica: flor.jimenez@ucr.ac.cr
}

Documento recibido: 23 de junio, 2014

Enviado a corrección: 15 de julio, 2014

Aprobado: 8 de diciembre, 2014 


\section{Introducción}

La disciplina de Orientación, posee la gran responsabilidad de aportar al fortalecimiento de la calidad de vida de la sociedad. Como una parte fundamental de la sociedad contemporánea, se encuentra la familia, permeada por los cambios tan acelerados que dicha sociedad experimenta. Producto de estos fenómenos, la familia se transforma constantemente y lucha por adaptarse a los requerimientos sociales, lo que la caracteriza como sistema viviente y en constante evolución.

Al respecto Walsh (2004) afirma que existe una configuración diversa de las familias producto de un contexto socio-histórico, donde destacan cuatro tendencias:

- Diversidad de las formas de familia

- Cambios en los roles de la familia

- Diversidad Cultural y disparidad socioeconómica

- Variedad y expansión del ciclo vital de la familia

Cuando se habla de la diversidad de formas de familia, se plantea que en la actualidad en la mayoría de las familias, se ha generado una proliferación de diversas estructuras familiares y redes de apoyo que reciben el nombre de configuraciones o formas no tradicionales de familia.

Esta, entre otras razones, hace necesaria la intervención orientadora, como un recurso valioso, que permita comprender la complejidad de los procesos familiares desde las interacciones, para favorecer las competencias familiares, a partir de las tareas propias del ciclo vital familiar. De esta manera, la Orientación cumple con una de sus principales funciones, acompañar en los procesos vitales de las personas y en sus tareas del desarrollo, como lo es la socialización en las familias, las cuales son parte de la comunidad educativa.

Para García y Martínez (2003) la Orientación Educativa es entendida como un proceso de ayuda a la persona, al grupo familiar y escolar. De ahí que se debe entender la familia y la escuela como contextos específicos de aprendizaje y socialización, que involucra a todos los miembros y subsistemas en que se organizan.

Es necesario que la Orientación apoye el acercamiento de las familias a los centros educativos de educación secundaria, debido a que se tienden a establecer diversas barreras que obstaculizan la relación entre familia e institución educativa, producto de la complejidad en las relaciones y de los sistemas sociales de las familias con hijas e hijos adolescentes. En este sentido, Davis y Lambie (2005) argumentan la importancia de que los centros educativos de secundaria participen a las familias en el proceso educativo y de diversas 
decisiones, como una poderosa influencia no sólo para mejorar el rendimiento académico del grupo de estudiantes, sino también para el desarrollo personal y social en general de las jóvenes y los jóvenes y de sus propias familias.

Además las personas profesionales en Orientación, con sus habilidades de liderazgo, conocimientos de los diferentes procesos del ciclo vital, entes activos y parte integral del programa educativo, pueden ser propiciadores de cambios sistémicos, al utilizar sus recursos para reducir las barreras que impidan por ejemplo, el éxito académico de las y los estudiantes, al fungir como mediadores en la relación escuela -hogar (Davis y Lambie, 2005).

Llama la atención que en los últimos informes de investigación que presenta el Estado de la Nación, la familia no ha sido un tema importante de estudio. La última información que se enfoca sobre ésta, data en el Estado de la Nación de 1996 que proporciona aspectos muy generales sobre este sistema, tales como los tipos de familia, violencia familiar y niñez y adolescencia, es decir, no se rescatan los cambios que los sistemas familiares costarricenses han experimentado durante los últimos 16 años ni cómo han incidido en la relación familia-escuela; situación que inquieta e insta a elaborar estudios relacionados que permitan actualizar y enriquecer lo existente hasta el momento.

Este estudio reviste importancia considerando que existen pocas investigaciones realizadas en el contexto nacional, que permitan describir la relación familia-escuela, desde un enfoque sistémico-ecológico.

Para efectos de esta investigación se considera la perspectiva ecológica, que según Corsi (2008), toma en cuenta los distintos contextos donde interaccionan las personas, para tener una visión integrada de la realidad a la que pertenecen las familias.

Es importante mencionar que los sistemas familiares están inmersos en exosistemas dentro de los cuales se identifican instituciones educativas, comunidades, ámbitos laborales, entre otros, que permean la estructura y el funcionamiento familiar. Estos exosistemas a su vez, están inmersos en macro sistemas, que son patrones más generalizados de la sociedad, como la cultura patriarcal, la idiosincrasia del país de origen o de un continente, entre otros.

De allí, radica la importancia de elaborar un estudio cuantitativo de tipo descriptivo, que permita analizar las necesidades de orientación que según las personas profesionales en Orientación manifiestan las madres y padres de familia de las instituciones donde laboran. Asimismo, se pretende identificar las inquietudes o necesidades que plantean las y los 
orientadores en lo referente al trabajo con madres y padres de familias.

\subsection{Objetivo general}

Analizar las necesidades de Orientación que tienen las madres y padres de familia según las personas profesionales en Orientación de los colegios académicos públicos de la Dirección Regional San José Norte.

\subsection{Objetivos específicos}

- Determinar las necesidades de Orientación de tipo familiar reconocidas por los y las profesionales en Orientación de los colegios académicos públicos de la Dirección Regional San José Norte.

- Determinar las necesidades de Orientación de tipo educativo relacionadas con el estudiantado que plantean madres y padres a los y las profesionales en Orientación de los colegios académicos públicos de la Dirección Regional San José Norte.

- Reconocer necesidades de capacitación sobre el trabajo con familias planteadas por las personas profesionales en Orientación de los colegios académicos públicos de la Dirección Regional San José Norte.

\section{Referente teórico}

Desde la visión sistémica de la familia, esta es vista de forma circular más que un pensamiento en líneas rectas o relaciones causa y efecto. Esta visión de la familia tiene su origen en una visión holística, donde todas las partes de un sistema están conectadas directa o indirectamente, de modo que al cambiar una de las partes el efecto se propaga a todas las demás. Esta forma de entender la familia da un acercamiento al fenómeno estudiado mucho más integral, porque permite visualizar todos los factores que intervienen en la complejidad de la cotidianidad familiar (Hernández, 1997).

Los sistemas familiares inician con la conformación de la pareja (parejas heterosexuales o parejas en situaciones especiales), la relación entre ambos es producto de intercambios repetidos: cada uno expresa sus particularidades como hábitos, creencias o necesidades que son intensificadas, anuladas o transformadas mediante la interacción. Así nace la unidad base de la familia, el inicio de la pareja (Belart y Ferrer, 1999).

Este patrón interaccional es único y particular en cada sistema y responde a las características de las personas que lo integran y depende de la manera en que estas se 
combinan, se complementan o se anulan entre sí. A este recién formado sistema se le denomina sub-sistema conyugal.

Con el nacimiento del primer hijo o hija el sistema familiar adquiere un subsistema, que cambia y modifica las interacciones del sistema conyugal asumiendo funciones parentales recién formadas, o sea el rol de papá, mamá y a su vez nace el subsistema filial o sea el subsistema de los hijos.

Cada individuo pertenece a diferentes subsistemas en los que posee diferentes niveles de poder y en los que aprende habilidades diferenciadas. Por ejemplo una mujer puede ser una hija, hermana mayor, hermana menor y así cumplir diferentes roles, de acuerdo con la estructura y dinámica familiar a la que pertenece. Esta estructura es el primer ente socializador que conoce el niño o la niña. Aquí aprende a desarrollar estrategias y habilidades básicas de interacción que posteriormente le servirán para incorporarse a una institución educativa.

La organización en subsistemas de una familia asegura el cumplimiento de las variadas funciones del sistema total, así como ofrece un campo diferenciado a cada miembro para ejercer sus habilidades interpersonales en distintos niveles (Szapazani y Hervís, 1985).

Este orden jerárquico que existe en el cual el niño o niña se incorpora desde su nacimiento, es el que le marca las pautas para aprender a relacionarse posteriormente con otros sistemas como por ejemplo el comunitario. Las personas pertenecen simultáneamente no sólo a varios subsistemas sino a diferentes, de esta pertenencia múltiple surge el intercambio de información entre un sistema y otro.

\subsection{Funciones del sub-sistema parental}

El sistema familiar está más protegido cuando sus integrantes de mayor madurez y experiencia vital, ejercen la autoridad. Cuando la autoridad la ejercen en familias monoparentales (solo mamá o solo papá) también es importante que exista claridad en el manejo de la autoridad, reglas, límites y clima de confianza.

Las madres y los padres tienen la capacidad y el poder de aplicar su exigencia, y son ellos mismos quienes establecen las reglas y límites de la familia. Para Glen (1992) existen tres tipos de estilos de paternidad y maternidad: autoritario, de firme autoridad y permisivo. Su definición se basa fundamentalmente en el equilibrio y el uso del poder entre padres e hijos. Los resultados de las evaluaciones indican que mamá y papá deben de ser firmes y 
capaces de controlar y exigir, al mismo tiempo afectuoso y dispuesto a aceptar a sus hijas e hijos y participar en su vida.

Los procesos de socialización van a implicar interacciones diarias entre padres, madres e hijos en contextos que diferirán en su función, en su estructura interpersonal y en su impacto en la hija y el hijo. A esto se le denomina ordenación jerárquica: no sólo comprende la autoridad que unos miembros ejercen sobre otros, las responsabilidades que asumen y las decisiones que toman, sino también la ayuda, protección, consuelo y cuidado que brindan a los demás.

Las madres y padres razonables y de autoridad firme producen más hijas e hijos plenamente competentes. Según Glen (1992) El principio fundamental de la paternidad razonable es el adecuado equilibrio entre las exigencias de los padres y su sensibilidad. El arte de ser madre o padre supone el uso de la conveniente cantidad de poder tanto para exigir como para detenerse en el momento preciso, siempre de acuerdo con las necesidades personales y el temperamento de los hijos.

Esta función de las madres y padres es fundamental en todas las etapas del desarrollo evolutivo porque este equilibrio homeostático (dinámico) entre el afecto, amor y autoridad, parental permea el comportamiento de la descendencia.

Las madres y padres son importantes, ya que suponen la principal fuente de referencia para el desarrollo de actitudes, creencias y valores de las personas adolescentes acerca de la vida en general y específicamente de su propio sentido del yo y su valía personal.

Musitu y Gutiérrez (1984) mencionan que es de suma importancia la interacción paterno-materno-filial basada en el apoyo y la cohesión o vinculación con la prole y en la expresión de la autoestima de la hija o el hijo y consecuentemente en su capacidad para adaptarse con facilidad a las diferentes situaciones, en su capacidad creativa y en su comportamiento.

Cuando las madres y padres aprenden a vincularse con las hijas e hijos de forma que se eduque a la prole manejando la disciplina en las etapas del ciclo vital familiar y adaptándose a las diferentes etapas de desarrollo evolutivo, la familia tiende a funcionar mejor.

Todas las familias tienen los recursos para generar cambios que faciliten su propio proceso de crecimiento familiar, pero en algunas ocasiones las familias necesitan de la ayuda del centro educativo favoreciendo procesos de socio-aprendizaje que faciliten el funcionamiento familiar. 


\subsection{Relación familia - centro educativo}

El conocimiento de lo que sucede en la familia y con ella es de gran importancia para el sistema escolar. La cohesión (vinculación que existe entre las partes del sistema) y flexibilidad (capacidad del sistema de ajustarse y autorregularse) del sistema familiar determina de alguna manera el estilo de relación que ha de mantener con la escuela. La familia es parte de la institución educativa y esta a su vez influye en esta.

Dabas (2003) indica que cada familia tiene una valoración particular de la escuela y por tanto varían sus expectativas con respecto al éxito de sus hijas e hijos, las demandas hacia el centro educativo elaboran diferentes maneras de enfrentar el fracaso escolar. Simultáneamente todos los miembros de la familia interaccionan en diversos contextos, modificando las posibilidades de aprendizaje de estos. Estas situaciones influencian y modifican las relaciones cotidianas.

Por otra parte el centro educativo representa el cambio y la transformación, considera Dabas (2003) que eso no ha cambiado, su esencia, a saber los elementos estructurales se han mantenido a lo largo del tiempo. La función del colegio como un sistema ha sido y es adaptar a las personas más jóvenes al mundo social vigente. Considera esta autora que esa función inicial de la escuela ha marcado su estructura y organización.

Posteriormente el quehacer educativo se valorizó en función del éxito y fracaso en la adquisición de aprendizajes y con ello surge el problema de la escuela como un problema de calidad, de ineficacia; es decir, lo que se planteaba era la inadaptación de la escuela a un mundo cambiante o bien la inadaptación de los estudiantes al sistema educativo. No se consideraban los cambios sociales y culturales.

Es importante tener claro que los cambios no solo provienen de la estructura macroeducativa. Madres padres y personal docente pueden construir un contexto significativo que permita compartir con respeto a los ámbitos de acción de cada uno. Las familias pueden compartir con las y los docentes modos de posicionarse y resolver los asuntos educativos de sus hijas e hijos y por otra parte puede favorecer a las personas docentes acciones que les permitan desarrollar un curriculum más ajustado al estudiantado.

En segundo lugar la participación. Esta decisión no puede ser tomada por solo la institución educativa, aunque puede ser la de la iniciativa; sin embargo, una vez que las familias responden se deben ajustar las metas y acciones considerando una nueva realidad. Esta aceptación no siempre es fácil que se dé. Debe ser una participación responsable que avance a la a la toma de decisiones compartida; pero se requiere de una nueva forma de 
pensar y claridad respecto a quién corresponde cada responsabilidad. El personal docente tiene la responsabilidad de definir los objetivos y la manera de implementarlos, y que pueda participar la familia.

En tercer lugar que familias y escuelas se abran para encontrar nuevas formas de conexión. Esto significa aceptar todos los saberes y la búsqueda activa entre todos los miembros de la red social, para que no se legitime únicamente el saber del docente. Si no que el padre y madre de familia se incorporen en el proceso de aprendizaje de sus hijas e hijos.

Pero no todas las instituciones educativas fomentan o están interesadas en esta articulación de la madre o padre al centro educativo, aunque diferentes investigaciones indican que esto influye directamente en el proceso de aprendizaje de jóvenes. Por el contrario, algunas veces la institución educativa desfavorece esta articulación, por falta de claridad de su función. Es importante formar redes sociales entre el centro educativo y las fuerzas vivas de las comunidades donde se están insertas para apoyar en la tarea educativa.

La red social implica un proceso de construcción de lo individual y lo colectivo. Es un sistema abierto que mediante un intercambio dinámico entre los integrantes de un colectivo (familia, equipo de trabajo, barrio, organización como la escuela, el hospital, etc.) y con integrantes de otros colectivos favorece la potencialización de los recursos que poseen y la creación de alternativas novedosas para la resolución de problemas o la satisfacción de necesidades (Dabas 2003).

Diversas investigaciones como la de Arguedas y Jiménez (2007) plantean la importancia de la familia en la permanencia u éxito las personas jóvenes en el sistema educativo. Al respecto mencionan:

Es importante señalar que la mayoría de las personas que han permanecido en la institución, han tenido el apoyo de sus madres o padres, los cuales asisten a reuniones, a entregas de notas o bien a cualquier convocatoria recibida por algún funcionario de la institución, tienen buen concepto de sus hijas e hijos y las o los respetan en sus diferencias (p. 26).

El éxito escolar y el fracaso escolar ya sea por razones de rendimiento académico, la deserción y la repitencia en la mayor parte de los casos, está relacionada con la falta de apoyo de la familia. Consideran que por lo general el sistema familiar asume un rol pasivo en su relación con el centro educativo y se recurre a este cuando se presentan problemas disciplinarios o bien de rendimiento académico. 
Al respecto Rodríguez (2009) plantea que el conocimiento de lo que sucede en la familia y con ella, es de gran importancia para el sistema escolar, porque es aquella la que, de alguna manera, determina el estilo de la relación que ha de mantener con la institución.

\subsection{La familia con hijos adolescentes}

Esta etapa significa para la persona adolescente el enfrentamiento de grandes desafíos, tales como, la pérdida y duelo del cuerpo de niña o niño, donde:

Al llegar a esta etapa la aparente tranquilidad que tenía el niño en etapas anteriores se pierde, y el adolescente se encuentra ante la necesidad de estructurar una nueva identidad personal, lo cual no es fácil ya que ha de integrar todas esas transformaciones. El cuerpo cobra gran importancia, es el punto de referencia en torno al cual se organiza la identidad y al mismo tiempo a través de él se relaciona con el mundo exterior (Belart y Ferrer, 1999 p. 152).

En general esta es la etapa del ciclo vital familiar que más se ha investigado por estar más que otra cargada de mitos y estereotipos; esta es una etapa de grandes retos y desafíos porque pone a las madres o padres a tener una relación diferente donde la persona adolescente quiere y lucha por encontrar quien es él o ella. $Y$ eso reta a sus madres y padres a estar de frente a sus propias preguntas existenciales. Las personas adolescentes establecen su autoestima principalmente en cualidades físicas, por eso para ellas y ellos es tan importante la apariencia física, la ropa, el peinado, entre otros.

Efectivamente, para la persona adolescente es prioritario afrontar la búsqueda de sí misma, es decir, saber quién es, qué es capaz de hacer y cuál es su papel en la sociedad. La adolescencia de las hijas e hijos puede ser también una forma indirecta de crecimiento para los padres, una experiencia enriquecedora puesto que dinamiza el sistema familiar y evita el anquilosamiento (Belart y Ferrer, 1999).

La adaptabilidad de las madres y padres para negociar, ceder y algunas veces ser firme, es el gran reto, la flexibilidad del sistema para ajustarse a las nuevas demandas de esta etapa del desarrollo evolutivo familiar va a ser fundamental. Así como la cohesión del sistema o sea la vinculación entre los miembros para que sea unida pero con la libertad de generar procesos de individuación esenciales en esta etapa.

Cuando la hija o el hijo presenta una crisis y el sub-sistema parental responde, ella o él confiará en ellos; estas pequeñas crisis son decisivas para las relaciones entre padres e hijas e hijos. Si los progenitores no le ayudan, no se enteran, le castigan o se ponen 
histéricos provocarán en él o ella sentimientos de culpa o de soledad que ensanchará la brecha generacional. Las madres y los padres tienen que estar a su disposición cuando la persona adolescente les necesite. Sin subestimar el problema o reaccionando de forma impulsiva o exagerada. Es fundamental que las hijas e hijos sepan que pueden contar con sus madres y padres en los momentos difíciles; si pueden confiar sin miedo a ser castigados representarán su mejor fuente de ayuda (Belart y Ferrer, 1999, p. 178).

Al respecto Hernández (1997) plantea que la persona adolescente no le va bien la rigidez ni la sobre protección. La rigidez ha de flexibilizarse mediante las negociaciones; si bien los padres han de asumir el control y marcar los límites, por su parte las hijas y los hijos han de tener "voz" para expresar sus opiniones. Sólo así se podrán llegar a acuerdos y soluciones satisfactorias para todas y todos. Es importante que queden claras las reglas a las que la persona adolescente tienen que atenerse en cuanto a sus estudios, horarios, obligaciones, dinero, etc., así como cuáles serán las conductas a seguir en caso de infracción a las reglas. En esta etapa ya no se trata de que los progenitores impongan las normas sino que han de ser pactadas conjuntamente entre padres e hijos e hijas.

\subsection{Orientación a las familias desde el centro educativo}

La Orientación a las familias cumple una función de entender la familia y el centro Educativo como contextos de aprendizaje y socialización en la que intervienen todos los actores educativos para generar estrategias que faciliten la dinámica familiar para prevenir, detectar dificultades, enfrentar y solucionar problemas. García y Martínez, (2003) plantean que la persona profesional en Orientación cumple una función fundamental como es ser ese agente de cambio social en la comunidad educativa. Lo que significa que su trabajo va dirigido a favorecer en sus orientadas y orientados las estrategias que aseguren la permanencia y el éxito académico de la población estudiantil. Estos mismos autores mencionan los principios que son ejes vertebrales de la función orientadora en el centro educativo:

1. El principio antropológico en el cual se ve al ser humano como libre de decidir, como ser individual y social, o sea que significa desarrollar estrategias para favorecer a la familia como única y particular.

2. Es el evaluativo lo que significa que el quehacer del profesional va precedido por una detección de necesidades de Orientación. 
3. Principio de intervención social o ecológico sistémico en el cual se centra en revisar las actuaciones en los diferentes contextos, donde cada estudiante interactúa.

Dentro del concepto de análisis de necesidades que el profesional efectúa del trabajo que realiza existen dos tipos de necesidades que plantea Sanz (1990, p. 76) "las primarias que residen en los sujetos - alumnos, alumnas, padres, madres, profesores y profesoras, que son los y las que reciben real o potencialmente los servicios diseñados en el programa. Y las necesidades secundarias que residen en la propia institución".

Otro aspecto importante es que siempre se debe tener claro que la familia tiene el derecho y deber de participar del proceso educativo de sus hijas e hijos. Y la institución educativa el deber de favorecer la relación familia centro educativo. $Y$ es función del profesional en Orientación facilitar el acercamiento centro educativo- familia.

\section{Procedimiento metodológico}

\subsection{Metodología}

\subsubsection{Tipo de investigación}

De acuerdo con las características de la presente investigación y sus propósitos, fue desarrollada bajo el enfoque cuantitativo. Se presenta una concepción global positivista, hipotética-deductiva, objetiva, particularista y orientada a los resultados. La función de la persona que investiga, consiste en ubicarse de forma externa al objeto que estudia (Barrantes, 2007).

Este estudio es de tipo no experimental, ya que se realiza de forma tal, que las variables no se manipularon deliberadamente. Lo que se pretendió en la investigación no experimental, es observar los fenómenos o situaciones tal y como se dan en su contexto natural, para después analizarlos. Es un estudio descriptivo (Hernández, Fernández y Baptista, 2010).

Asimismo, con lo propuesto se analizó las necesidades de Orientación que presentaban las madres y padres de familia y que han sido detectadas por las personas profesionales en Orientación de la Dirección Regional de Educación San José-Norte. Al tener una visión global y generalizada sobre la temática en estudio, se lograron analizar las necesidades detectadas, así como, proponer algunas recomendaciones dirigidas a las instituciones educativas, en relación a la puesta en práctica de algunas acciones que permitan la atención de las madres y padres. 
Esa es la razón por la que se eligió un estudio de tipo descriptivo, en el cual el propósito es relatar situaciones y eventos, tal y como lo afirma (Hernández et. al., 2010, p. 60) "Los estudios descriptivos buscan especificar las propiedades importantes de personas, grupos, comunidades o cualquier fenómeno que sea sometido a análisis. Mide o evalúan diversos aspectos, dimensiones o componentes del fenómeno a investigar."

\subsubsection{Problema de investigación}

Mediante el proceso investigativo, se pretendió responder al siguiente problema de investigación: ¿Cuáles son las necesidades de Orientación de madres y padres detectadas por las personas profesionales en Orientación de instituciones de educación secundaria académica diurna públicas de la Dirección Regional Norte?

\subsubsection{Sujetos de investigación}

El universo lo constituyen 35 personas profesionales en Orientación de los colegios académicos diurnos públicos de la Dirección Regional de Educación San José Norte. Se trabajó con toda la población.

\subsubsection{Definición de variables}

\subsubsection{Definición conceptual}

Necesidades de Orientación de tipo familiar: Se refiere a la discrepancia entre el cumplimiento de las tareas parentales como son el cuido, la protección y el establecimiento de reglas y límites y la expectativa del centro educativo respecto a cumplimiento de estas (Belart y Ferrer, 1999; Hernández ,1997).

Necesidades de Orientación de tipo educativo: Se refiere a la discrepancia entre el apoyo que consideran las y los profesionales de Orientación deberían dar madres y padres en cuanto a las condiciones que facilitan el proceso educativo de sus hijas e hijos. Incluyendo la participación e involucramiento de madres y padres en las actividades del centro educativo, el apoyo emocional en su rol de estudiante (Belart y Ferrer, 1999; Hernández ,1997).

\subsubsection{Definición operacional}

Para la presente investigación las variables operacionales fueron entendidas igual que las conceptuales. 


\subsubsection{Instrumento}

Con el propósito de obtener la información de este estudio, se utilizó la técnica de cuestionario. El cual está constituido por preguntas abiertas (13) de los temas a investigar y fue sometido a criterio de profesionales en Orientación en ejercicio de colegios diurnos, que no formaban parte del grupo investigado.

Después de recopilar la información, las investigadoras se dieron a la tarea de leerla e identificar categorías de análisis que dieran respuesta a las necesidades de tipo familiar y educativo. Se ubicó la información recogida en cada una de las categorías, según lo mencionado por cada persona participante. Se agrupo la información que concordaba y se cuantifico las frecuencias. Lo anterior permitió elaborar cuadros y gráficos con la información recopilada.

\section{Información recopilada y discusión de resultados}

A continuación se presenta la información recopilada, así como, su análisis.

Se observa en el Gráfico 1 que las personas participantes del estudio declaran que las necesidades básicas del estudiantado no están siendo cubiertas en su totalidad por parte de las madres y padres de familia. Dos personas consideran que las necesidades básicas de salud, educación, acceso a vivienda y capacidad económica para cubrir las necesidades mínimas, no están siendo satisfechas por las madres y padres. Por ejemplo, la mayoría de la población estudiantil debe solicitar beca de comedor en el colegio y en las instituciones del estado beca económica.

Se ve que las madres y los padres no pueden cubrir necesidades básicas de sus hijas e hijos, tales como: comida y vivienda (la mayoría viven en casas alquiladas con problemas de construcción), con mucha dificultad podrían cubrir necesidades de otro orden tales como las modas, actividades recreativas, entre otras (Belart y Ferrer, 1999).

También señalan las personas que la mayoría de las familias están conformadas por madre e hijos o hijas, y que muchas de ellas trabajan en labores domésticas que no les permite cubrir las necesidades básicas del estudiantado. Al trabajar la mayoría de las mujeres en trabajos poco remunerados; desde una visión eco sistémica de la familia, esto tiene una influencia en el micro sistema o sistema familiar de no cubrir necesidades de orden básico (Corsi, 2008; Walsh, 2004), lo cual llevaría a las personas adolescentes a adaptarse a lo que el sistema les está brindando. Las personas profesionales en Orientación mencionan que lo que si se observa es que varias madres y padres dan apoyo, cuido y vigilancia a sus 
hijas e hijos. Pero que están más centrados en resolver los problemas económicos que los afectivos.

\section{Gráfico 1}

Satisfacción de las necesidades básicas del estudiantado de los colegios diurnos públicos de la Dirección Regional de Educación San José Norte por parte de las madres y padres de familia según los profesionales en Orientación, 2013

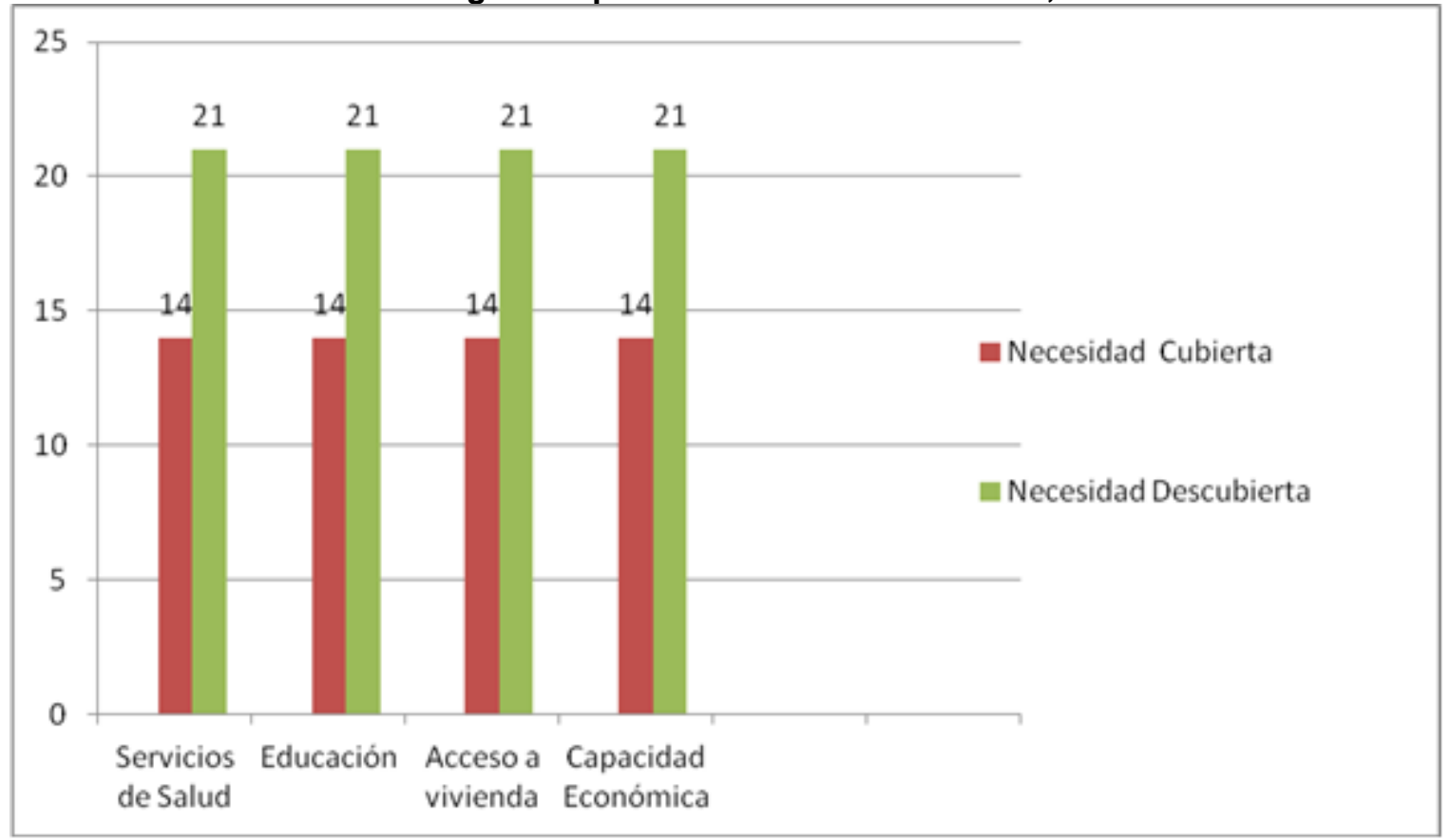

Fuente: Elaboración propia

Se nota en el Gráfico 2 que veinte y ocho de las personas profesionales en Orientación que se entrevistaron consideran que las madres y padres de familia no satisfacen las necesidades básicas de sus hijos e hijas, según lo esperado por la institución. Sin embargo, no se puede dejar de lado que al pertenecer la mayoría del estudiantado de las instituciones que abarcaron el estudio a una clase baja, no se podría pensar en que las madres y padres no estén queriendo satisfacer las necesidades básicas de sus hijos e hijas, sino más bien que la capacidad económica no permite, ni facilita a las personas responsables del estudiantado satisfacerlas. 
Gráfico 2

Opinión de los profesionales en Orientación de los colegios diurnos públicos de la Dirección Regional de Educación San José Norte sobre la satisfacción de necesidades básicas que realizan las madres y padres de familia según lo esperado por la institución, 2013

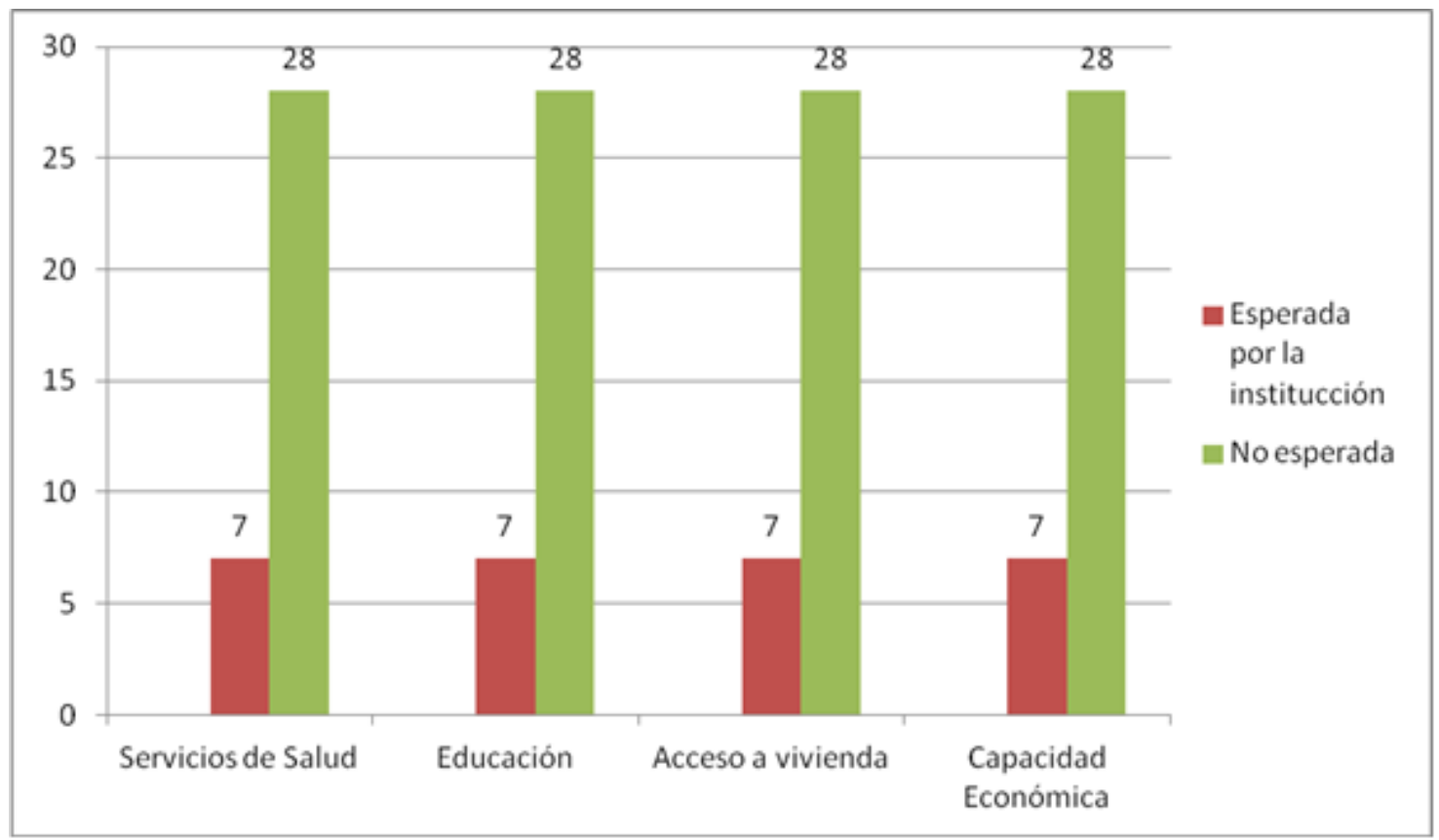

Fuente: Elaboración propia

Lo que sí consideran las personas participantes del estudio que podrían satisfacer las madres y padres de familia y no lo hacen en su mayoría, es el tener reglas claras en la familia, tener más supervisión y cuido de los hijos e hijas y acompañarles más en su proceso de enseñanza y aprendizaje. Es importante que las madres y padres consideren que si bien no es sano la rigidez excesiva, ni la sobreprotección, ya que no ayuda a tener límites claros, lo cual es parte fundamental para que se genera la independencia y autonomía de las personas adolescentes (Hernández, 1997).

Al observar las respuestas obtenidas de las personas participantes del estudio sobre si las familias del estudiantado tienen reglas claras y explícitas; veinte y una de las personas señalan que no. Sin embargo, lo más importante a rescatar es que los profesionales en Orientación mencionan que las madres y padres no saben cómo implementar las normas, muchas veces las ponen en práctica por imposición y no de forma consensuada. Algunas de las personas profesionales en Orientación declaran que varias madres y padres de familia esperan que la institución educativa establezca los límites y la verificación de los mismos en la institución, y a su vez, esto repercute en la dinámica familiar. 
Es necesario que si bien los Departamentos de Orientación contribuyen en asesorar a las madres y padres sobre la práctica de reglas claras y poner consecuencias. La mayoría de las madres y padres como bien lo señalan los participantes no cuentan con las competencias o herramientas para lograrlo. Sin embargo es oportuno tomar en cuenta que los padres tienen el poder y responsabilidad de establecer reglas firmes, de autoridad y permisivas a la vez (Glen, 1992).

\section{Gráfico 3}

Presencia de reglas claras y explícitas en las familias según las personas profesionales en Orientación de los colegios diurnos públicos de la Dirección Regional de Educación San José Norte, 2013

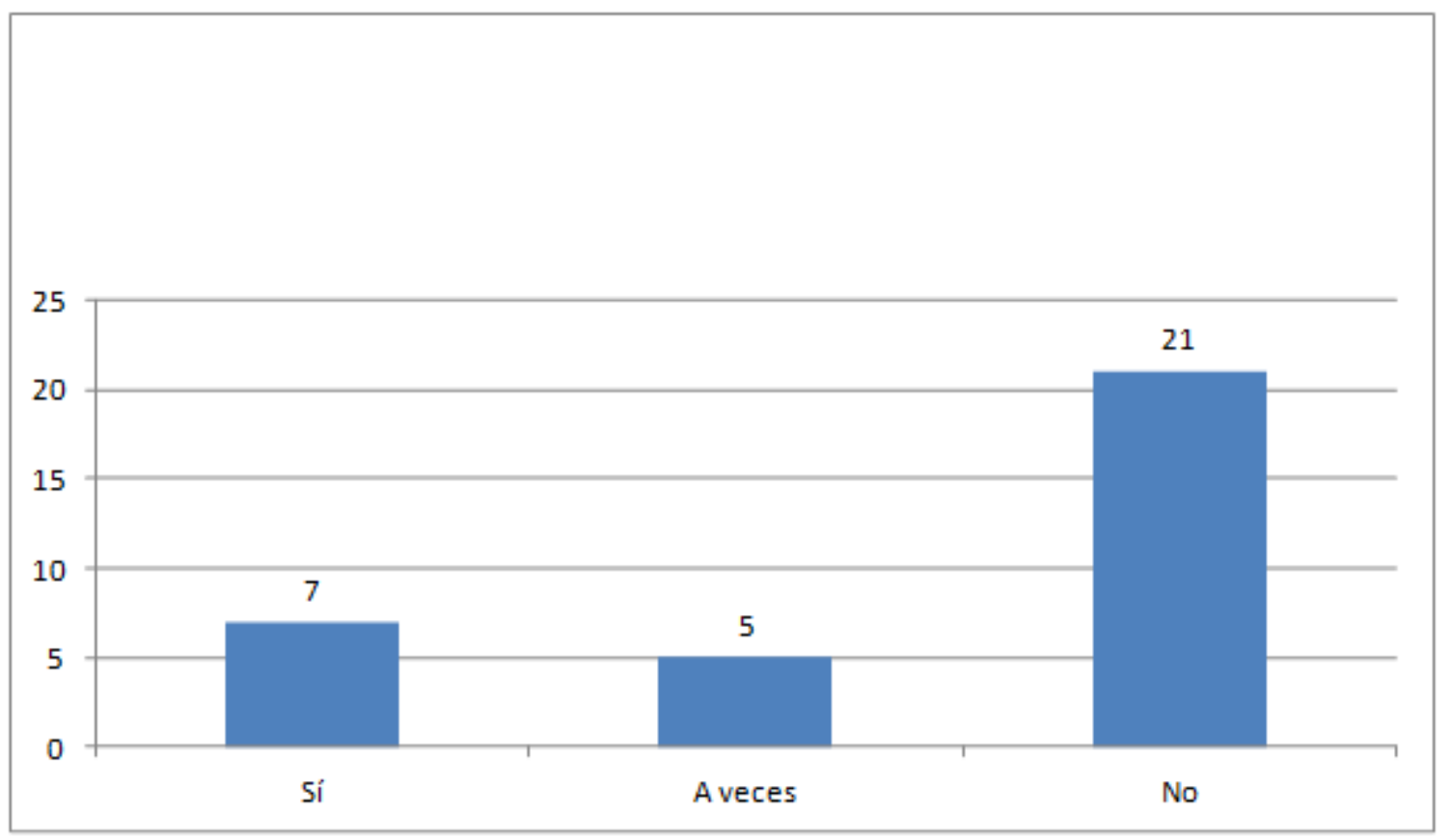

Fuente: Elaboración propia

Se observa en el Gráfico 4 que las madres y padres de familia asisten mayoritariamente para conversar con el personal docente o administrativo. Sin embargo, las personas profesionales en Orientación que respondieron a esta pregunta, declaran que cuando las madres y padres visitan la institución en la mayoría de las veces es porque se les cita y no por iniciativa propia.

Es importante resaltar aquí que las personas entrevistadas señalan que en sétimo año es cuando más madres y padres asisten a la institución, lo cual se podría deber a que como vienen de la escuela necesitan adaptarse. 


\section{Gráfico 4}

Acciones que según las personas profesionales en Orientación de los colegios diurnos públicos de la Dirección Regional de Educación San José Norte realizan las madres y padres de familia para favorecer el proceso educativo de su hija o hijo, 2013

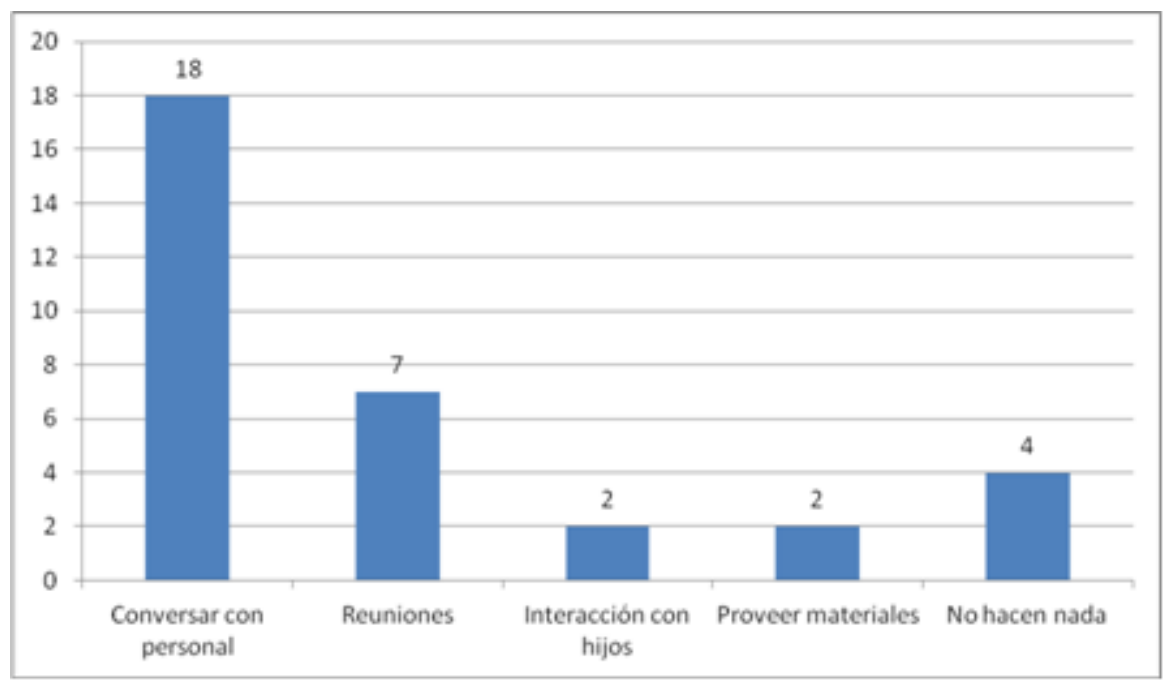

Fuente: Elaboración propia

Las personas profesionales en Orientación (20) señalan que la asistencia de las madres y padres a la institución no es la esperada y (15) consideran que si es la esperada. Sin embargo, anotan que las madres y padres deberían tener mayor compromiso y tener una mayor participación. Es importante que las madres y padres de familia según las personas entrevistadas deberían asumir su papel en el proceso de enseñanza y aprendizaje de sus hijos e hijas. Diversas investigaciones como la Davis y Lambie (2005) mencionan que es necesario que en los departamentos de Orientación apoyen el acercamiento de las familias a los centros educativos, para la toma de diversas decisiones que influyen en distintos aspectos como el rendimiento académico del estudiantado, así como, en desarrollo personal de las y los jóvenes y las propias familias. 


\section{Gráfico 5}

Opinión de las personas profesionales en Orientación de los colegios diurnos públicos de la Dirección Regional de Educación San José Norte sobre si las acciones que realizan las madres y padres de familia para favorecer el proceso educativo de su hija o hijo son las esperadas por la institución, 2013

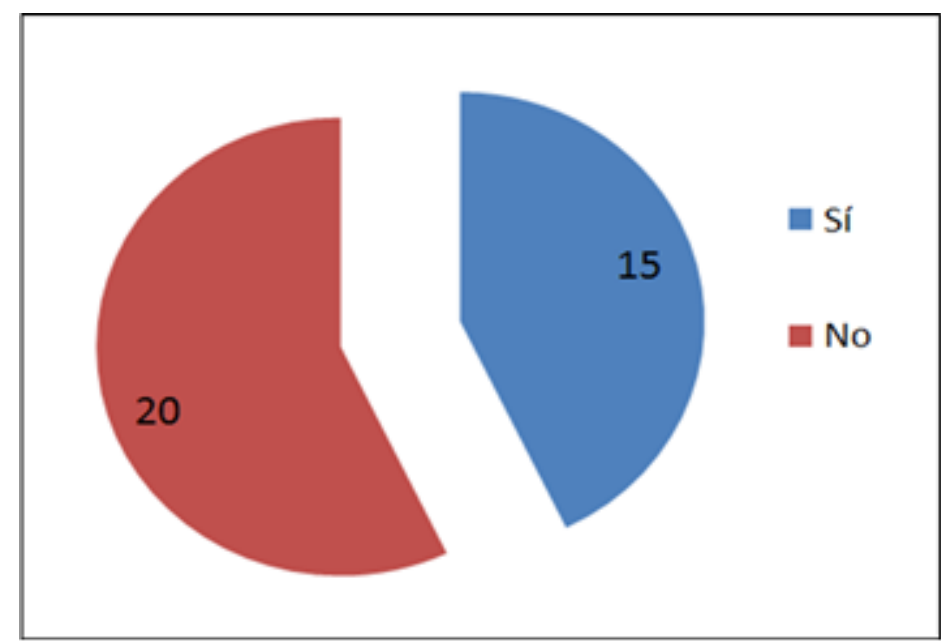

Fuente: Elaboración propia

Se observa en el Gráfico 6 que las personas profesionales en Orientación señalan diferentes acciones que se realizan en la institución para apoyar a las madres y padres de familia en su quehacer para la formación del estudiantado.

De las acciones que se llevan a cabo en la institución, algunas de ellas son talleres de diferentes temáticas, talleres recreativos y reuniones para entrega de notas.

Se podría inferir que las instituciones que forman parte de esta investigación no cuentan con estrategias o acciones que estén motivando a madres y padres a asistir. Las y los profesionales en Orientación con sus habilidades de liderazgo, conocimiento de los procesos familiares y el ciclo vital familiar, como entes activos y parte integral del proceso educativo, deben ser propiciadores de cambios, utilizando los recursos para reducir las barreras que impiden el éxito escolar de los y las estudiantes y fungir como mediadores y promotores de la relación escuela-hogar (Davis y Lambie, 2005). 


\section{Gráfico 6}

Acciones que según las personas profesionales en Orientación de los colegios diurnos públicos de la Dirección Regional de Educación San José Norte realiza la institución dirigidas a las madres y padres de familia, 2013

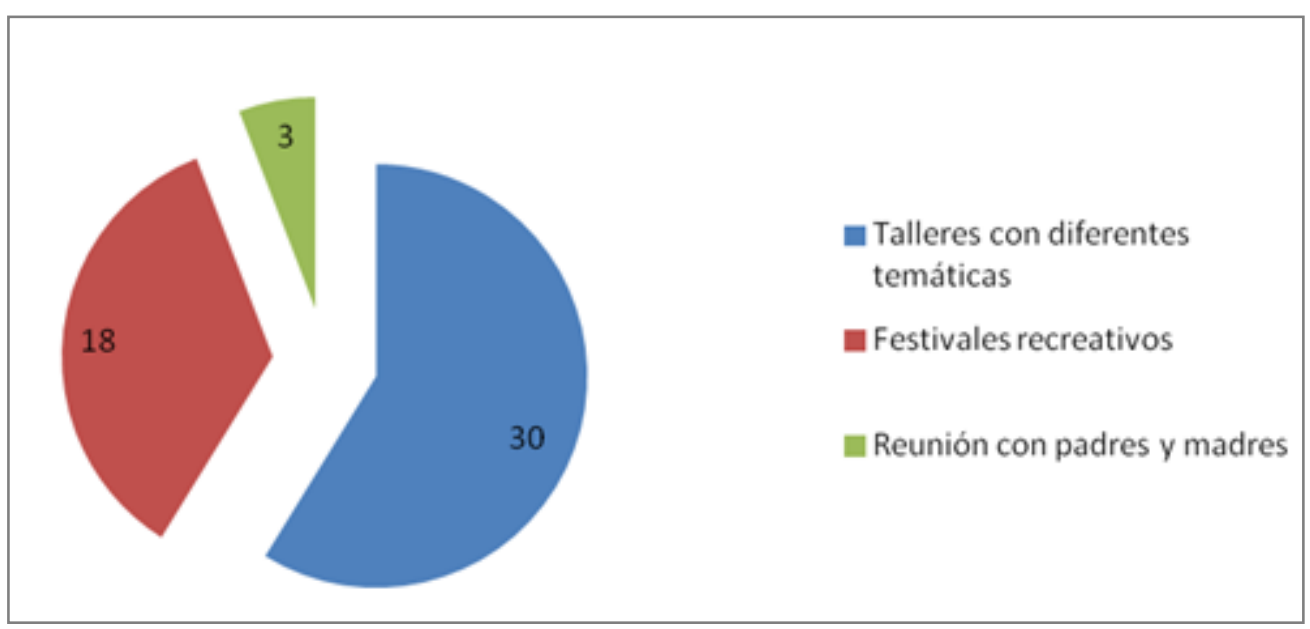

Fuente: Elaboración propia

En el Gráfico 7 se observa que según las personas del estudio las instituciones no están satisfechas con la participación de las madres y padres de familia en las actividades que se promueven. Declaran que las madres y padres mencionan que no asisten debido a que no tienen tiempo para eso. En el momento que se observa un poco más de participación es cuando se hace la entrega de notas o actividades de orden recreativo. Algunas personas participantes concluyen que hay poco compromiso para con los hijos e hijas, así como, poca identificación con la institución.

También declaran que la participación de las madres y padres se presenta más en sétimo que en los otros niveles. Como lo habíamos mencionado al parecer las madres y padres consideran importante apoyar y acompañar al estudiantado en la transición entre la escuela y el colegio.

Algunas de las personas participantes del estudio mencionan que usan el correo electrónico para comunicarse con las madres y padres. 


\section{Gráfico 7}

Opinión de las personas profesionales en Orientación de los colegios diurnos públicos de la Dirección Regional de Educación San José Norte sobre la satisfacción o insatisfacción institucional del involucramiento de las madres y padres de familia en las actividades que promueve la institución, 2013

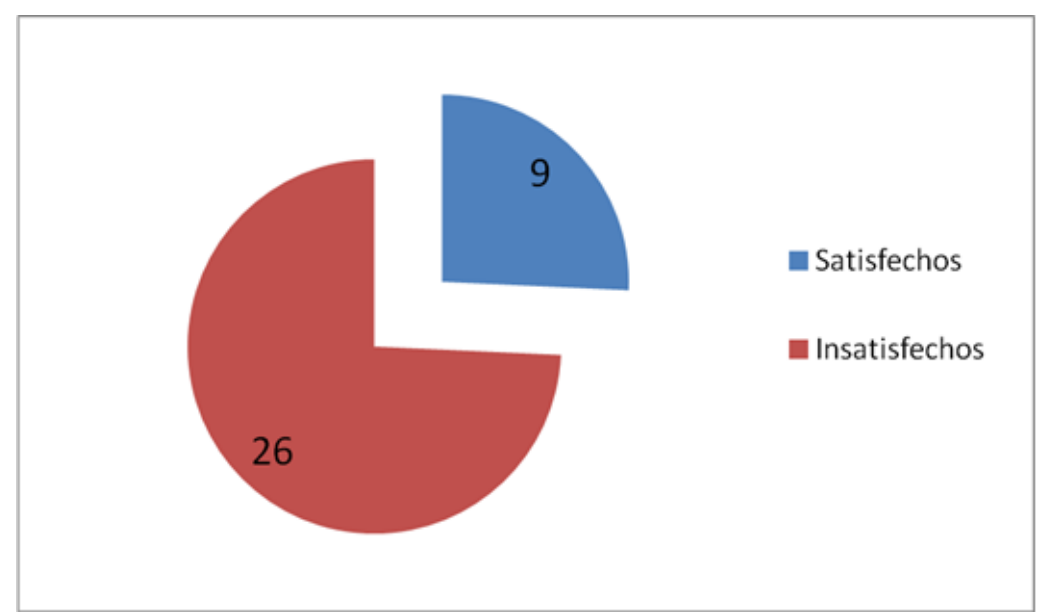

Fuente: Elaboración propia

Se observa en el Gráfico 8 que las personas del estudio señalan que las madres y los padres casi nunca apoyan emocionalmente al estudiantado. Asimismo, opinan que en varias oportunidades no les acompañan en su etapa del desarrollo y en las tareas académicas. También o que no dan la importancia adecuada al estudiantado en los problemas académicos o emocionales que presentan.

También declaran que es importante que las madres y padres conozcan los cambios de las personas adolescentes, ya que en muchos momentos algunas madres y padres maltratan a las hijas y los hijos por situaciones que son características propias del desarrollo evolutivo.

Además se menciona que varios padres o madres exigen notas a sus hijos e hijas, pero no apoyan para que el estudiantado logre conseguirlas. Unido a esto, está el que las madres y los padres casi nunca llegan a la institución, situación que hace más complicado el logro académico del estudiantado. Lo anterior provoca bajas notas, unidas a los índices altos de pobreza del estudiantado.

La responsabilidad de las madres y padres no solo tiene que ver con el poner reglas hacia su prole, sino también con la ayuda, protección, consuelo y cuidado de estos. Las madres y padres razonables y de firma autoridad promueven hijos e hijas plenamente competentes (Glen, 1992). 


\section{Gráfico 8}

Opinión de las personas profesionales en Orientación de los colegios diurnos públicos de la Dirección Regional de Educación San José Norte sobre el apoyo emocional que recibe el estudiantado por parte de las madres y padres de familia, 2013

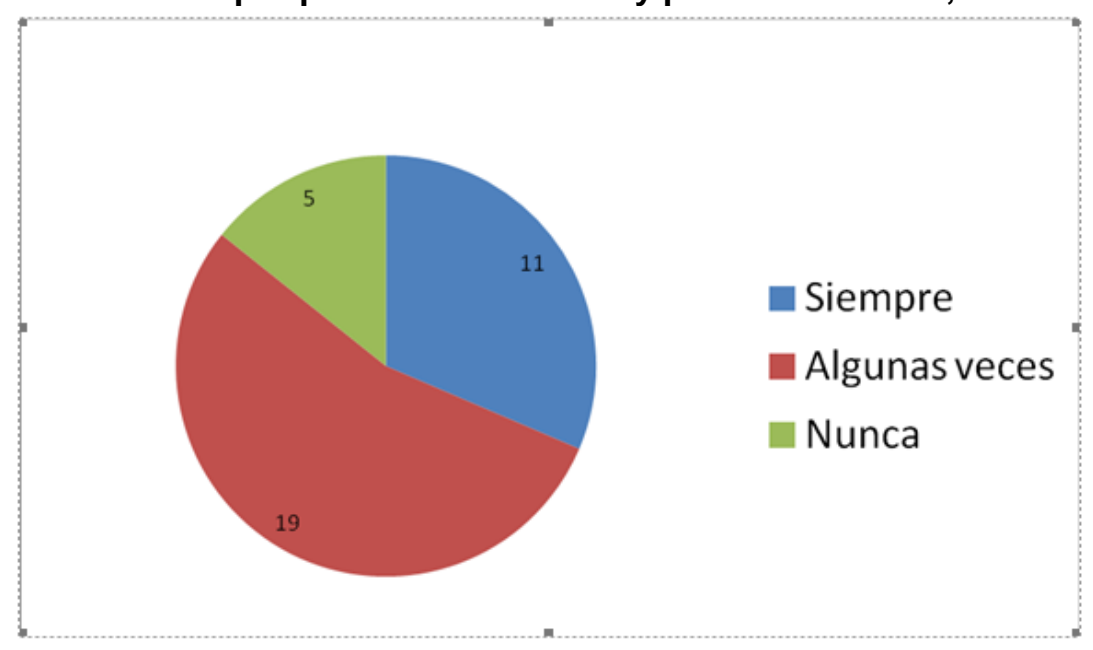

Fuente: Elaboración propia

En el Gráfico 9 se nota que las personas participantes del estudio opinan que las madres y padres de familia no están apoyando emocionalmente a sus hijas y sus hijos como lo esperaría la institución.

Las personas entrevistadas señalan que hace falta que en las instituciones se implementen escuelas para madres y padres, ya que las personas progenitoras no cuentan con las herramientas (comunicación, manejo de límites, entre otros) necesarias para poder apoyar emocionalmente y académicamente a sus hijos y sus hijas.

Algunas de las personas participantes señalan que en varios momentos se han tenido que referir madres y padres al Patronato de la Infancia. Lo anterior debido a que hay familias que presentan violencia doméstica, no tienen trabajo estable y algunos miembros de algunas familias tiene problemas de drogadicción. 


\section{Gráfico 9}

Opinión de las personas profesionales en Orientación de los colegios diurnos públicos de la

Dirección Regional de Educación San José Norte sobre si el apoyo emocional que recibe el estudiantado por parte de las madres y padres de familia es el esperado por la institución, 2013

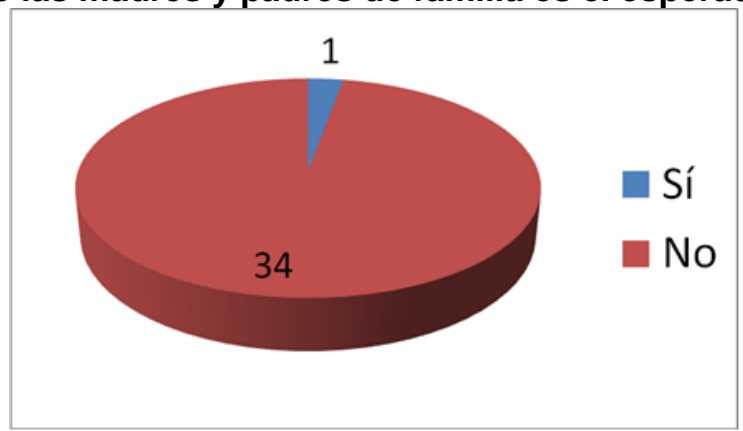

Fuente: Elaboración propia

Como se observa en el Gráfico 10 las personas participantes del estudio declaran que las necesidades de Orientación que ellos y ellas observan en las madres y padres de familia son: manejo de límites (12) comunicación (11) y sentido de vida (10). Estos temas son identificados por la mayoría de las personas del estudio. Es oportuno que la institución liderada por la persona profesional en Orientación puede promover el acercamiento centro educativo-familia y generar una cultura institucional que favorezca en las personas participantes el desarrollo de destrezas en relación con las temáticas de interés de las madres y padres.

\section{Gráfico 10}

Necesidades de Orientación identificadas por las personas profesionales en Orientación de los colegios diurnos públicos de la Dirección Regional de Educación San José Norte que deben ser trabajadas con madres y padres, 2013

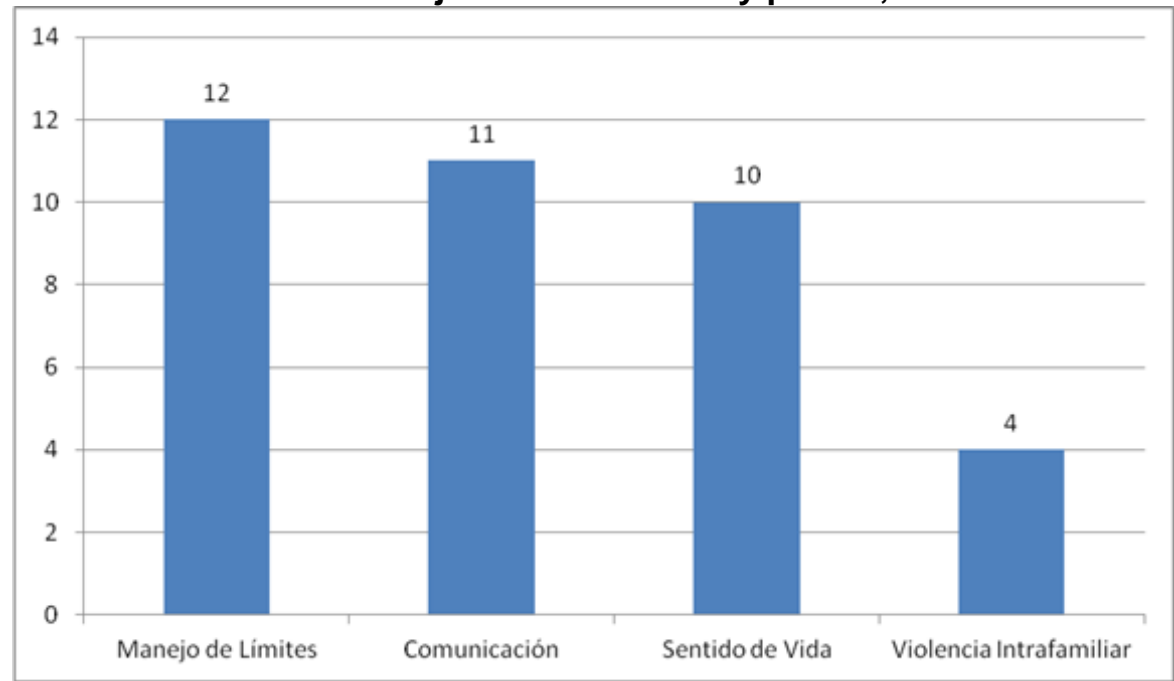

Fuente: Elaboración propia 
Se observa en el Gráfico 11 las personas profesionales en Orientación sienten una necesidad de capacitación en trabajo con familia y sentido de vida.

\section{Gráfico 11}

Necesidades de capacitación identificadas por las personas profesionales en Orientación de los colegios diurnos públicos de la Dirección Regional de Educación San José Norte, 2013

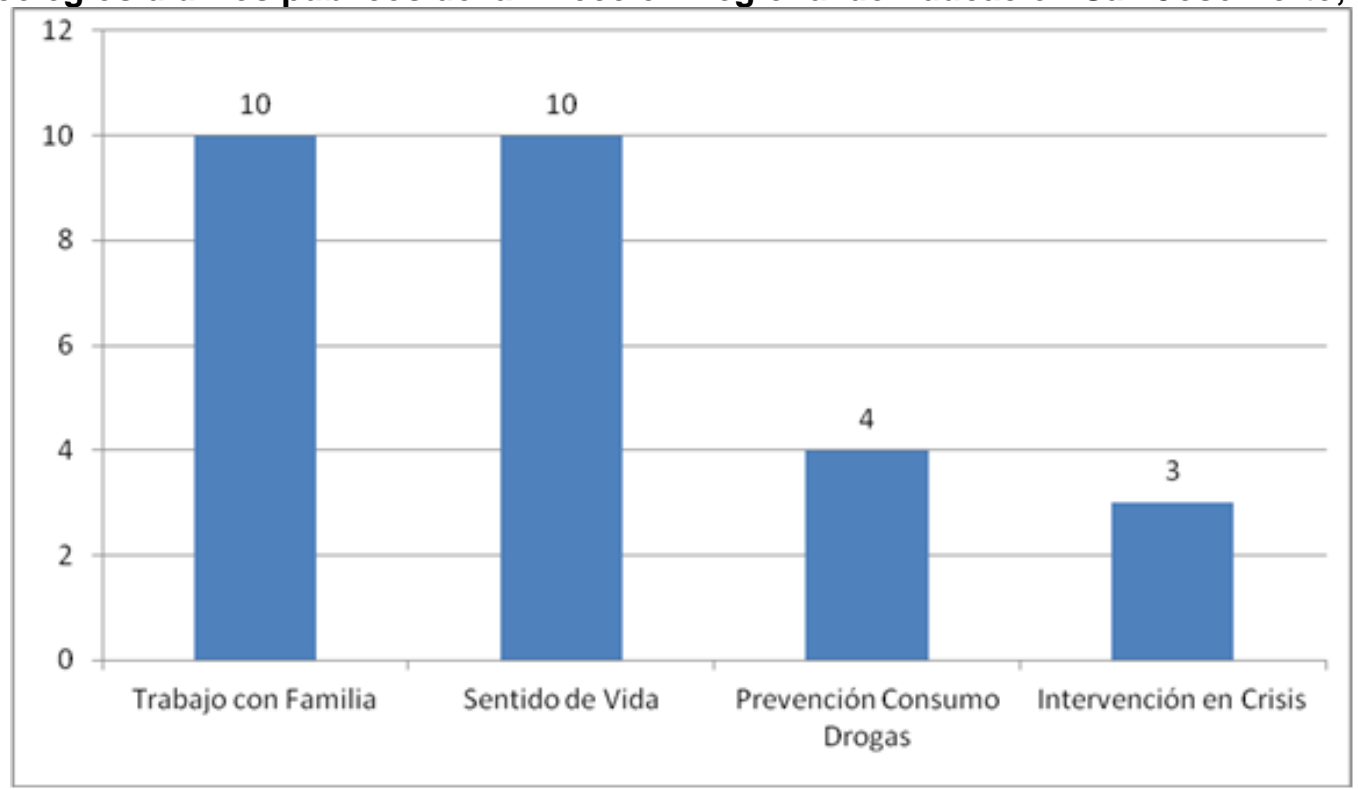

Fuente: Elaboración propia

\section{Conclusiones}

No hay diferencias significativas en lo referente al apoyo académico y emocional que reciben los estudiantes y las estudiantes durante la secundaria. Se observa que hay más acompañamiento en sétimo año. Pueden existir múltiples razones que expliquen esta realidad, una de estas, podría ser que al ser sétimo una etapa de transición escuela-colegio, las madres y padres sientan la necesidad de acompañar a su hija o hijo en este periodo.

Las personas profesionales en Orientación entrevistadas tienen claro que si bien algunos madres y padres desean satisfacer las necesidades básicas de sus hijos y sus hijas, por situaciones de pobreza, falta de empleo, violencia doméstica, entre otros, no se logra.

Las personas profesionales en Orientación plantean la necesidad de recibir capacitación en el trabajo con familias y sentido de vida prioritariamente.

Las instituciones educativas favorezcan el acercamiento de madres y padres de familia a la institución, por medio de citas, en donde son convocados por la institución por problemas que presentan las y los estudiantes, pocas veces asisten a la institución por iniciativa propia. Sería oportuno la implementación de diferentes estrategias (escuela para 
padres, visita a las familias, entre otros) que promuevan la relación institución educativa y madres y padres.

Las madres y padres de familia esperan que sea la institución educativa la que establezca los límites y la verificación de los mismos, y que esto favorezca la convivencia familiar.

Al centro educativo le preocupa el involucramiento de las madres y padres. Sin embargo, no promueven políticas claras para ese involucramiento.

Algunas de las personas participantes señalan que usan las tecnologías de la información para comunicarse con las madres y padres de familia.

Las madres y padres de familia señalan los participantes del estudio que necesitan formación en lo referente a manejo de límites y consecuencias, y reglas claras.

Las personas profesionales en Orientación plantean la necesidad de recibir capacitación en el trabajo con familias y sentido de vida prioritariamente.

\section{Recomendaciones}

Es necesario que las instituciones educativas promuevan una cultura de acercamiento de madres y padres de familia al proceso educativo del estudiantado. Teniendo esta el favorecimiento escolar de las y los estudiantes.

Promover desde el Departamento de Orientación del Ministerio de Educación Pública o la Escuela de Orientación y Educación Especial propuestas dirigidas a las personas profesionales en Orientación para que a partir de capacitaciones sobre la implementación y el trabajo en escuela de padres, se puedan realizar, ya sea institucionalmente o bien por comunidades educativas.

Que la Universidad de Costa Rica por medio del Programa de Educación Continua de la Escuela de Orientación y Educación Especial ofrezcan: cursos, seminarios, charlas entre otros, sobre temáticas relacionadas con la familia y sentido de vida de las mismas; que proporcione herramientas teórico prácticas que favorezcan al ser realizadas, la relación centro educativo-familia.

\section{Agradecimientos}

Agradecimientos a las personas profesionales de los Departamentos de Orientación de los colegios académicos diurnos de la Dirección Regional San José Norte y a la Directora Regional de la Dirección Regional San José Norte. 


\section{Referencias}

Arguedas Negrini, Irma y Jiménez Segura, Flor (2007). Factores que promueven la Permanencia de Estudiantes en la Educación Secundaria. Revista INIE, 7(3), 1-36. Recuperado de http://revista.inie.ucr.ac.cr/uploads/tx magazine/fact.pdf

Barrantes Echeverría, Rodrigo. (2007). Investigación un camino al conocimiento. San José, C.R.: EUNED.

Belart, Ascensión y Ferrer, María. (1999). El ciclo de la Vida: Visión Sistémica de la familia. Bilbao, España: Serendipity.

Corsi, Jorge. (2008). Violencia familiar: una mirada interdisciplinaria sobre un grave problema social. Buenos Aires, Argentina: Paidós.

Dabas, Elina. (2003). Redes Sociales, familias y Escuela. Buenos Aires, Argentina: Paidós.

Davis, Keith y Lambie, Glenn. (2005). Family Engagement: a collaborative, systemic approach for middle school counselors. Professional School Counseling, 9(2), 144151.

García Mediavilla, Luis y Martínez González, María de Codés. (2003). Orientación educativa en la familia y en la escuela. Madrid, España: Dykinson.

Glen, Austin. (1992). Amor y Poder entre padres e hijos, Como hacer nuestros hijos Capaces y seguros de sí mismos. México: Editorial Patria.

Hernández Córdoba, Angela. (1997). Familia, Ciclo Vital y Psicoterapia Sistémica Breve. Buenos Aires, Argentina: Editorial El Buho.

Hernández Sampieri, Roberto, Fernández Collado, Carlos y Baptista Lucio, Pilar. (2010). Metodología de la investigación. México: McGraw Hill.

Musito, Gonzalo, Román, José y Gutiérrez, Melchor. (1984). Educación Familiar y socialización de los hijos. Barcelona, España: Idea.

Rodríguez, Marta Verónica. (2009). Familia escuela. Resiliencia familiar. Buenos Aires, Argentina: Dunken.

Sanz Oro, Rafael. (1990). Evaluación de programas de Orientación Educativa. Madrid, España: Pirámide.

Szapazani, Leonor y Harvey, Frank. (1985). Terapia Familiar Sistémica. Material mimeográfico. San José, Costa Rica: Hospital Nacional Psiquiátrico de Costa Rica.

Walsh, Froma. (2004). Resiliencia Familiar. Argentina: Amorrortu. 